\section{Pupil dilation using drops vs gel: a comparative study}

E Moisseiev, D Loberman'1, E Zunz, A Kesler, A Loewenstein and J Mandelblum

\begin{abstract}
Purpose To compare the efficacy in pupil dilation and degree of discomfort between topical instillation of mydriatic drops and gel. Methods The study included 60 patients with no previous ocular history of trauma and surgery. One eye was dilated with two drops (tropicamide $\mathbf{0 . 5 \%}$ and phenylephrine $10 \%$ ), and the other with one drop of gel (tropicamide $0.5 \%+$ phenylephrine $5 \%$ ). Pupil size was measured by a Colvard pupillometer at baseline and 5, 15, 30, and $45 \mathrm{~min}$ following instillation. Pain upon instillation was measured by visual analog scale (VAS).

Results There was no difference in pupil size at baseline. Use of the gel achieved greater mydriasis than drops $(P=0.01)$, and was also associated with lower pain scores $(P=0.003)$. In diabetic patients, pupil size was smaller at baseline and following instillation of drops and gel. Use of the gel achieved an even greater degree of pupil dilation in this subset of patients than drops $(P=0.019)$.

Conclusions Gel formulation achieved significantly greater pupil dilation than drops, despite a lower concentration of phenylephrine, and was also associated with significantly lower patient discomfort. This study is the first report of improved mydriatic efficacy in diabetic patients.

Eye (2015) 29, 815-819; doi:10.1038/eye.2015.47; published online 10 April 2015
\end{abstract}

\section{Introduction}

Cataract is the leading cause of age-related blindness worldwide, ${ }^{1}$ and cataract extraction is the most commonly performed ophthalmic surgery. ${ }^{2}$ An adequately dilated pupil is a prerequisite for safe cataract extraction surgery, and small pupil size has been shown to be associated with increased risk for intraoperative and early postoperative complications. ${ }^{3-5}$

Preoperative pupil dilation is commonly achieved by repeated administration of mydriatic drops. This process is time consuming, and it has also been shown that repeated instillation of drops may damage the corneal epithelium, which may cause patient discomfort and interfere with the visibility during surgery. ${ }^{6}$ Therefore, additional methods for pupil dilation have been explored. Intracameral injection of mydriatic agents has been shown to be safe and effective, ${ }^{7,8}$ but was reported to be inferior to conventional topical mydriasis in several studies. ${ }^{9,10}$ Use of wicks saturated in mydriatic agents has been shown to be comparable to use of drops, but has been associated with an increased risk of conjunctival and corneal abrasions. 6,11,12 One recent study has reported the topical use of a gel containing phenylephrine, tropicamide, diclofenac, and lidocaine for topical anesthesia and mydriasis, which was found to achieve greater and more rapid pupil dilation compared with drops. It has been suggested that the greater efficacy is owing to the fact that the gel was retained in close proximity to the eye while the drops were cleared by the lacrimal system, and that the gel formulation provided additional permeability through the cornea. ${ }^{13}$

The purpose of our study was to compare the efficacy in pupil dilation between topical instillation of mydriatic drops and gel, and to compare the degree of patient discomfort during both methods of mydriasis.

\section{Material and methods}

\section{Patient selection}

All patients in this study were 18 years or older, and were recruited during their visit at our clinic. Exclusion criteria included any prior ocular trauma or surgery, any ocular condition requiring treatment by intravitreal injections, any ocular condition that affects pupillary function (such as optic neuropathy, Adie's tonic pupil, oculomotor nerve palsy, and so on), and any use of drops or gels. Male patients were also specifically asked about current or previous use of $\alpha 1$-adrenergic receptor antagonists (such as tamsulosin) for prostate problems, and were excluded if their
Department of

Ophthalmology, Tel Aviv Sourasky Medical Center, Affiliated to the Sackler Faculty of Medicine, Tel Aviv University, Tel Aviv, Israel

Correspondence: E Moisseiev, Department of Ophthalmology, Tel Aviv Sourasky Medical Center, 6 Weitzman Street, Tel Aviv 64239, Israel Tel: +972 3 6973408; Fax: +972 36973870 .

E-mail: elad_moi@ netvision.net.il

${ }^{1}$ Equal contribution as first author.

Received: 16 October 2014 Accepted in revised form: 23 February 2015 Published online: 10 April 2015

This work was performed in partial fulfillment of the M.D. thesis requirements of the Sackler Faculty of Medicine, Tel Aviv University. 
history was positive for their use. Patients with pseudoexfoliation or anisocoria $>0.5 \mathrm{~mm}$ at baseline were also excluded. Patients with known allergy to tropicamide or phenylephrine were not included. The study protocol was reviewed and approved by the Institutional Review Board, and a written informed consent was obtained from all participants. All applicable institutional and governmental regulations concerning the ethical use of human volunteers were followed during this research.

Recorded parameters included demographic information and previous medical and ocular history. Specifically, patients were asked whether they had diabetes mellitus or not, and this was confirmed with their medical records.

\section{Pupil dilation and measurement}

Each participant received one drop of tropicamide $0.5 \%$ (Mydramide, Fischer Pharmaceutical Labs., Tel Aviv, Israel) and one drop of phenylephrine hydrochloride $10 \%$ (Efrin, Fischer Pharmaceutical Labs., Tel Aviv, Israel) in one eye, and one drop of a gel containing tropicamide $0.5 \%$ and phenylephrine $5 \%$ in the other. Drops were administered to both eyes within $30 \mathrm{~s}$ of each other, by a technician, in a single-masked manner.

We note that the gel was designed especially for this study, and is not a commercially available product. The gel was manufactured by a GMP approved pharmacy under sterile conditions (Concept pharmaceutics, KfarSaba, Israel). After compounding, the gel was distributed into individual small tubes, for single use with each patient. Tubes were not reused in order to avoid any variation in the gel's efficacy due to different times after their opening. This study did not evaluate the gel's shelf life after its opening.

Horizontal pupil diameter was measured by a Colvard pupillometer (Oasis Medical, London, England), at baseline, and at 5, 15, 30, and $45 \mathrm{~min}$ following pupil dilation. All measurements were made by a single observer (DL), who was masked for the method of mydriasis used on all eyes, in the same room under photopic conditions (luminance of 5.0 candelas $\left(\mathrm{cd} / \mathrm{m}^{2}\right)$ ).

\section{Pain measurement}

Pain was measured by subjective grading on a visual analog scale (VAS), immediately following the instillation of pupil dilation drops and gel. The VAS is a horizontal line measuring exactly $10 \mathrm{~cm}(100 \mathrm{~mm})$, as shown in Figure 1. Each patient was asked to mark a vertical line crossing the horizontal line, according to his or her subjective pain assessment during the drops and gel instillation, ranging from no pain at all to maximal pain. The distance between the left edge of the horizontal line and the vertical mark made by the patient was later measured and recorded

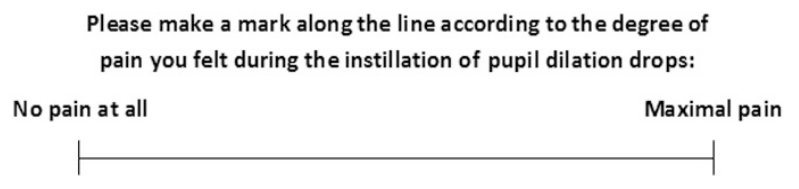

Figure 1 An example of the VAS graded by the participant at each time point in the study. All lines were exactly $10 \mathrm{~cm}$ in length.

in $\mathrm{mm}$, and transformed into a score between 0 and 100 . All VAS measurements were collected by the same observer (DL), after explaining this method to the patients. The VAS is a common tool for assessing pain and other symptoms, which has been shown to be a valid and reliable research method in previous clinical studies. ${ }^{14-17}$ It has been successfully used in ophthalmological studies evaluating pain associated with ocular surgery, intravitreal injections and topical therapies. ${ }^{18-25}$

\section{Statistical analysis}

Correlations between continuous variables were analyzed using Pearson's correlation coefficient, and paired $t$-tests were used to analyze associations between categorical parameters. An analysis of variance (ANOVA) with repeated measure (over time) was performed in order to assess the change in pupil diameter over time. The statistical significance level was set at 0.05. Data were analyzed using SPSS for windows version 20. (SPSS Inc., Chicago, IL, USA).

\section{Results}

The study included 60 patients, $37(61.7 \%)$ women and 23 $(38.3 \%)$ men. Mean age was $66.6 \pm 17.9$ years (range 19-93 years). Seventeen $(28.3 \%)$ patients were diabetic.

\section{Comparison of pupil dilation between drops and gel}

Pupil diameter at baseline was not significantly different between eyes that received drops and eyes that received gel. After instillation of the drops and gel, pupil diameter was enlarged. ANOVA with repeated measures over time comparing pupil diameter between eyes treated with drops and gel demonstrated a significantly larger pupil diameter in eyes that received gel for pupil dilation $(P=0.01)$. The mean difference in final pupil diameter at $45 \mathrm{~min}$ was $\sim 0.2 \mathrm{~mm}$ (Figure 2). Pupil size data at all time points is provided in Table 1 .

\section{Comparison of pain measurements between drops and gel}

Mean pain scores in eyes that received drops were $33.6 \pm 25.6$, and $23.5 \pm 26.3$ in eyes that received gel. 
This difference was statistically significant between groups $(P=0.003)$, with less pain associated with use of the gel.

\section{Effect in diabetic patients}

Pupil diameter at baseline was $3.6 \pm 0.9 \mathrm{~mm}$ (median $3.75 \mathrm{~mm}$ ) in diabetic patients, and $4.16 \pm 0.9 \mathrm{~mm}$ (median $4 \mathrm{~mm}$ ) in non-diabetics. This difference was statistically significant $(P=0.01)$. Regardless of pupil dilation method, pupil diameter was significantly smaller in eyes of diabetic patients at all time points (Figure 3).

The effect of the drops and gel on pupil dilation were analyzed separately in diabetic patients. Significantly larger pupil diameter was achieved in eyes that received gel than in eyes that received drops $(P=0.019)$. The mean difference in final pupil diameter at $45 \mathrm{~min}$ was $\sim 0.3 \mathrm{~mm}$ (Figure 4). Pain scores were also significantly lower in eyes treated with gel in this subset of patients $(P=0.04)$.

\section{Discussion}

Our results demonstrate that use of a gel containing tropicamide $0.5 \%$ and phenylephrine $5 \%$ achieved significantly greater pupil dilation than use of tropicamide $0.5 \%$ and phenylephrine $10 \%$ drops. These results support those of the previous study comparing gel and drops for pupil dilation. ${ }^{13}$ It should be noted that the previous study was smaller and included only 20 patients, and that it compared a gel containing



Figure 2 Comparison of pupil diameter (mean \pm SE) between eyes that received drops and eyes that received gel for pupil dilation. tropicamide $1 \%$ and phenylephrine $10 \%$ with tropicamide $1 \%$, phenylephrine $2.5 \%$, and cyclopentholate $1 \%$ drops. This study included a larger cohort, and the fact that the phenylephrine concentration was lower in the gel than in the drops supports the hypothesis that using a gel formulation will achieve greater mydriasis. As previously mentioned, there are two possible explanations for this finding. First, the gel is more viscous than the drops and is not rapidly cleared by the lacrimal system, and therefore stays as a depot on the ocular surface and may have a longer duration of action. Second, the biochemical properties of the gel may give it improved corneal penetrance and increase its efficacy.

In addition, this study is the first to compare the patients' perceived level of pain between instillation of drops and gel. Our results indicate that the gel was associated with significantly lower pain scores than drops. There are two possible explanations for this difference. First, eyes dilated with drops received two drops that contain a preservative (both Mydramide and Efrin drops contain benzalkonium chloride), whereas eyes dilated with gel received one drop of a preservative-free formulation. It has been shown that preservative-free formulations are associated with lower pain score than the equivalent preserved formulations..$^{20,21,26}$ Second, it is possible that the difference resulted from the increased osmolality of the phenylephrine 10\% drops (Osmolality values were for the tropicamide $0.5 \%$ drops, phenylephrine $10 \%$ drops and the gel were 308, 1000, and 590 mosmol, respectively). Increased osmolality has been associated with ocular discomfort, most notably in dry eye syndrome, and use of drops with lower osmolality has been correlated with improved tolerability. ${ }^{27-29}$

A novel finding in this study is that significantly better mydriasis was achieved by the gel compared with the drops in diabetic patients. The difference in this subset of patients was larger and more significant than in nondiabetic patients. At baseline, pupil diameter was significantly smaller in diabetic patients $(P=0.01)$, which is compatible with previous reports of smaller pupil size and reduced response to pharmacological mydriasis in diabetes. ${ }^{30-32}$ Our results indicate that use of the gel achieves greater pupil dilation than drops in diabetic patients. It is possible that the suggested mechanisms for the higher efficacy of the gel in general, the gel's longer duration of action on the ocular surface, and increased

Table 1 Pupil size data at all time points of the study

\begin{tabular}{llllll}
\hline Groups & \multicolumn{1}{c}{ Baseline } & \multicolumn{1}{c}{5 min } & \multicolumn{1}{c}{15 min } & \multicolumn{1}{c}{$30 \min$} & $45 \mathrm{~min}$ \\
\hline Drops & $4.01 \pm 0.91(4)$ & $4.66 \pm 1.06(5)$ & $6.30 \pm 1.05(6.25)$ & $7.10 \pm 1.02(7)$ & $7.47 \pm 1.01(7.5)$ \\
Gel & $4.04 \pm 0.93(4)$ & $4.64 \pm 1.04(4.75)$ & $6.26 \pm 1.04(6.25)$ & $7.25 \pm 1.05(7.5)$ & $7.66 \pm 1.02(8)$ \\
\hline
\end{tabular}

Values are provided as mean $\pm \mathrm{SD}$ (median), in $\mathrm{mm}$. 



Figure 3 Comparison of pupil size (mean \pm SE) between diabetic and non-diabetic patients (a -eyes that received drops, $b$ - eyes that received gel). Pupil diameter was significantly smaller in eyes of diabetic patients at all time points.



Figure 4 Comparison of pupil diameter $($ mean \pm SE) in diabetic patients between eyes that received drops and eyes that received gel for pupil dilation.

penetrance, are even more significant in eyes of diabetic patients who tend to have smaller pupils.

A limitation of this study is its relatively small sample size. However, we note that the series is much larger than that reported in the only previous study on pupil dilation using a gel, which included only 20 patients, ${ }^{13}$ and that its size was still sufficient to achieve statistically significant results. Another limitation is that patients' eyes were not examined for conjunctival and corneal irritation following instillation of the drops and gel. This parameter may have correlated with the VAS pain scores. In addition, the concentration of phenylephrine was different between the drops and gel (10\% and 5\%, respectively). However, it should be noted that the gel was found to have better efficacy despite the lower concentration, which supports that hypothesis that a gel formulation for pupil dilation will be advantageous over drops.

In conclusion, the use of gel was demonstrated to achieve greater mydriasis than drops, supporting the finding of the previous smaller study. ${ }^{13}$ The superior efficacy was even more pronounced in eyes of diabetic patients, and this is the first report of a pharmacological delivery method that improves pupil dilation in these patients. In addition, use of the gel was also associated with lower pain scores, implying better patient tolerability to it than drops. A single instillation of the gel achieved greater pupil dilation with less discomfort, and appears to be a better alternative than the commonly used drops. This method may improve pupil dilation before cataract surgery, as well as other ocular procedures, dilated fundus examinations and imaging techniques such as fluorescein angiography and optical coherence tomography. Such a gel may have a widespread use in the current practice of ophthalmology.

\section{Summary}

What was known before

- Preoperative pupil dilation is most commonly achieved by repeated instillation of mydriatic drops.

- Only one small study had previously compared the efficacy of pupil dilation between drops and gel, and demonstrated that gel achieved greater mydriasis.

\section{What this study adds}

- This study includes a larger cohort of patients, and its results show that gel is more effective than drops in pupil dilation. This supports and strengthens the only previous study on this issue.

- A novel finding that gel is even more significantly effective in pupil dilation than drops in diabetic patients, who tend to have smaller pupils and be more resistant to dilation.

- A novel finding that use of a gel for pupil dilation is associated with lower pain scores (ie, better tolerability) than drops.

- The results of this study indicate that a pupil dilation gel may be a better alternative for preoperative preparation of patients, and may have an important place in the clinical practice of ophthalmology. 


\section{Conflict of interest}

The authors declare no conflict of interest.

\section{References}

1 Thylefors B, Negrel AD, Pararajasegaram R, Dadzie KY. Global data on blindness. Bull World Health Organ 1995; 73: $115-121$.

2 Rutkow I. Surgical operations in the United States: then (1983) and now (1994). Arch Surg 1997; 132(9): 983-990.

3 Lumme P, Laatikainen LT. Risk factors for intraoperative and early postoperative complications in extracapsular cataract surgery. Eur J Ophthalmol 1994; 4: 151-158.

4 Guzek JP, Holm M, Cotter JB, Cameron JA, Rademaker WJ, Wissinger $\mathrm{DH}$ et al. Risk factors for intraoperative complications in 1000 extracapsular cataract cases. Ophthalmology 1987; 94: 461-466.

5 Vasavada A, Singh R. Phacoemulsification in eyes with a small pupil. J Cataract Refract Surg 2000; 26: 1210-1218.

6 Sengupta S, Subramoney K, Srinivasan R, Nongrum B, Agarwal V, Pandian DG et al. Use of a mydriatic cocktail with a wick for preoperative mydriasis in cataract surgery: a prospective randomised controlled trial. Eye (Lond) 2010; 24: $118-122$.

7 Mori Y, Miyai T, Kagaya F, Nagai N, Osakabe Y, Miyata K, Amano S. Intraopertaive mydriasis by intracameral injection of mydriatic eye drops: in vivo efficacy and in vitro safety. Clin Experiment Ophthalmol 2011; 39: 456-461.

8 Gupta SK, Kumar A, Agarwal S, Agarwal S. Phacoemulsification without preoperative topical mydriatics: induction and sustainability of mydriasis with intracameral mydriatic solution. Indian J Ophthalmol 2014; 62: 333-336.

9 Lundberg B, Behndig A. Intracameral mydriatics in phacoemulsification cataract surgery. J Cataract Refract Surg 2003; 29: 2366-2371.

10 Morgado G, Barros P, Martins J, Lima A, Martins N. Comparative study of mydriasis in cataract surgery: topical versus Mydrasert versus intracameral mydriasis in cataract surgery. Eur J Ophthalmol 2010; 20: 989-993.

11 Ong-Tone L. Use of a wick to deliver preoperative mydriatics for cataract surgery. J Cataract Refract Surg 2003; 29: 2060-2062.

12 Dubois V, Wittles N, Lamont M, Madge S, Luck J. Randomised controlled single-blind study of conventional versus depot mydriatic drug delivery prior to cataract surgery. BMC Ophthalmol 2006; 6: 36.

13 Jinapriya D, Almeida DRP, Johnson D, Irrcher I, El-Defrawy SR. Anasthetic plus dilating gel improves dilation for cataract surgery. Can J Ophthalmol 2012; 47: 145-149.

14 Woods CA, Cumming B. The impact of test medium on use of visual analogue scales. Eye Contact Lens 2009; 35: 6-10.

15 Johnson C. Measuring pain. Visual analog scale versus numeric pain scale: what is the difference? J Chiropr Med 2005; 4: 43-44.

16 Breivik EK, Bjornson GA, Skovlund E. A comparison of pain rating scales by sampling from clinical data. Clin J Pain 2000; 16: $22-28$.

17 Salo D, Eget D, Lavery RF, Garner L, Bernstein S, Tandon K. Can patients accurately read a visual analog pain scale? Am J Emerg Med 2003; 21: 515-519.
18 Jacobi PC, Dietlein TS, Bacobi FK. A comparative study of topical vs retrobulbar anesthesia in complicated cataract surgery. Arch Ophthalmol 2000; 118: 1037-1043.

19 Crandall AS, Zabriskie NA, Patel BC, Burns TA, Mamalis N, Malmquist-Carter LA et al. A comparison of patient comfort during cataract surgery with topical anesthesia and intracameral lidocaine. Ophthalmology 1999; 106: 60-66.

20 Chiambaretta F, Creuzot-Garcher C, Pilon F, Pouliquen P, Rebika H, Dubray C et al. [Ocular tolerance of a new formulation of nonpreserved diclofenac]. J Fr Ophthalmol 2004; 27: 739-744.

21 Moisseiev E, Varssano D. Comparison of ocular tolerability between preserved and preservative-free diclofenac sodium drops. J Ocul Pharmacol Ther 2011; 27: 333-337.

22 Moisseiev E, Regenbogen M, Bartfeld Y, Barak A. Evaluation of pain in intravitreal bevacizumab injections. Curr Eye Res 2012; 37: 813-817.

23 Yau GL, Jackman CS, Hooper PL, Sheidow TG. Intravitreal injection anesthesia - Comparison of different topical agents: A prospective randomized controlled trial. Am J Ophthalmol 2011; 151: 333-337.

24 Cintra LP, Lucena LR, Da Silva JA, Costa RA, Scott IU, Jorge R. Comparative study of analgesic effectiveness using three different anesthetic techniques for intravitreal injection of bevacizumab. Ophthalmic Surg Lasers Imaging 2009; 40: 13-18.

25 LaHood BR, Sherwood D, Suter A. Comparative assessment of the effectiveness of anaesthesia for intravitreal bevacizumab injection. Clin Exp Ophthalmol 2011; 39: 184-185.

26 Rouland JF, Traverso CE, Stalmans I, Fekih LE, Delval L, Renault $\mathrm{D}$ et al. Efficacy and safety of preservative-free latanoprost eyedrop, compared with BAK-preserved latanoprost in patients with ocular hypertension or glaucoma. Br J Ophthalmol 2013; 97: 196-200.

27 McGinnigle S, Naroo SA, Eperjesi F. Evaluation of dry eye. Surv Ophthalmol 2012; 57: 293-316.

28 Parra A, Gonzalez-Gonzales O, Gallar J, Belmonte C. Tear fluid hyperosmolality increases nerve impulse activity of cold thermoreceptor endings of the cornea. Pain 2014; 155(8): 1481-1491.

29 Stahl U, Wilcox M, Stapleton F. Role of hypo-osmotic saline drops in ocular comfort during contact lens wear. Cont Lens Anterior Eye 2010; 33: 68-75.

30 Karavanaki K, Davies AG, Hunt LP, Morgan MH, Baum JD. Pupil size in diabetes. Arch Dis Child 1994; 71: 511-515.

31 Hayashi K, Hayashi H. Pupil size before and after phacoemulsification in nondiabetic and diabetic patients. J Cataract Refract Surg 2004; 30: 2543-2550.

32 Lei HL, Yang KJ, Sun CC, Chen CH, Huang BY, Ng SC et al. Obtained mydriasis in long-term type 2 diabetic patients. J Ocul Pharmacol Ther 2011; 27: 599-602.

(c) (1) () $\ominus$ This work is licensed under a Creative Commons Attribution-NonCommercialNoDerivs 4.0 International License. The images or other third party material in this article are included in the article's Creative Commons license, unless indicated otherwise in the credit line; if the material is not included under the Creative Commons license, users will need to obtain permission from the license holder to reproduce the material. To view a copy of this license, visit http://creativecommons.org/licenses/by-nc-nd/4.0/ 